\title{
Evaluation of Extracellular Matrix Formation in Polycaprolactone and Starch-Compounded Polycaprolactone Nanofiber Meshes When Seeded with Bovine Articular Chondrocytes
}

\author{
Marta Alves da Silva, M.Sc., ${ }^{1-3}$ Aileen Crawford, Ph.D., ${ }^{3}$ Jenifer Mundy, B.S., ${ }^{3}$ Albino Martins, B.S., ${ }^{1,2}$ \\ Jose V. Araújo, M.Sc., ${ }^{1,2}$ Paul V. Hatton, Ph.D., ${ }^{3}$ Rui L. Reis, Ph.D., ${ }^{1,2}$ and Nuno M. Neves, Ph.D. ${ }^{1,2}$
}

Cartilage defects are a major health problem. Tissue engineering has developed different strategies and several biomaterial morphologies, including natural-based ones, for repairing these defects. We used electrospun polycaprolactone (PCL) and starch-compounded PCL (SPCL) nanofiber meshes to evaluate extracellular matrix (ECM) formation by bovine articular chondrocytes (BACs). The main aim of this work was to evaluate the suitability of PCL and SPCL nanofiber meshes in chondrocyte cultures, and their capability to produce ECM when seeded onto these nanostructured materials. The effect of culture conditions (static vs dynamic) on ECM formation was also assessed. BACs were seeded onto PCL and SPCL nanofiber meshes using a dynamic cellseeding procedure and cultured under static or dynamic conditions for 4 weeks. Constructs were characterized using scanning electron microscopy, histology, immunolocalization of collagen types I and II, and glycosaminoglycan (GAG) quantification. Results show an extensive cell colonization of the entire nanofiber mesh, for both materials, and that chondrocytes presented typical spherical morphology. Some degree of cell infiltration inside the nanofiber meshes was noticeable for both materials. ECM formation and GAG were detected throughout the materials, evidencing typical construct maturation. PCL and SPCL nanofiber meshes are suitable as supports for ECM formation and therefore are adequate for cartilage tissue-engineering approaches.

\section{Introduction}

$\mathbf{J}_{\mathrm{h}}^{\mathrm{o}}$ OINT DISEASES due to cartilage degeneration are a major health problem for people of all ages, but particularly for elderly people. Osteoarthritis, for example, affects $80 \%$ of people aged 60 and older. One therapeutic option is nonsteroidal antiinflammatory drug treatment aimed at the delay and control of cartilage loss. ${ }^{1}$ Other options involve surgical procedures, such as arthroplasty with implantation of joint prosthesis. ${ }^{2}$ These procedures are able to improve joint function considerably, although the patients' mobility may be limited, and some problems at the interface of bone and implant may arise, causing the need for revision. ${ }^{2} \mathrm{Al}-$ though surgical outcome is good initially, durability of solutions and improvement of patients' quality of life is far from ideal. Tissue engineering represents an alternative route to current treatments. Its strategy may be based in cell injections into the defect and fixation with a membrane ${ }^{3}$ or implantation of combinations of cells, scaffolds, and growth factors ${ }^{4}$ that may result in extracallular matrix (ECM) production.

Articular hyaline cartilage is an avascular tissue that covers the joint and acts as a load-bearing and wear-control structure. Because cartilage is subjected to mechanical stimulus in its natural environment, a strategy to regenerate cartilage under the action of similar stimulus is typically followed. It has been shown in vitro that the culture environment greatly affects chondrocyte proliferation in a three-dimensional scaffold. ${ }^{5}$ Bioreactors, including spinner-flasks, rotating-wall vessels, and direct perfusion, are designed to provide a controlled environment for tissue-engineered cartilage production. ${ }^{6}$ Biomaterial morphology, ${ }^{7}$ as well as culture conditions and cell source, ${ }^{8}$ can also influence the quality of tissueengineered cartilage constructs.

Biodegradable biomaterials, either natural or synthetic, have been processed into scaffolds for tissue engineering. ${ }^{9,10}$ Polycaprolactone (PCL) is a biodegradable polymer that belongs to the aliphatic polyester family. Currently, this class of

\footnotetext{
${ }^{1}$ 3Bs Research Group-Biomaterials, Biodegradables, and Biomimetics, University of Minho, Avepark, Guimarães, Portugal.

${ }^{2}$ Institute for Biotechnology and Bioengineering, PT Associated Laboratory, Braga, Portugal.

${ }^{3}$ Centre for Biomaterials and Tissue Engineering, School of Clinical Dentistry, Claremont Crescent, University of Sheffield, Sheffield, United Kingdom.
} 
materials is among the most attractive polymers that meet various medical and physical demands for safe clinical applications. PCL is used in clinical practice as a biomaterial because of its good mechanical properties; its ability to form compatible blends and copolymers with a wide range of other polymers, including natural-based polymers; low toxicity; biocompatibility; and biodegradability. ${ }^{11-13}$ In addition, its composition with starch offers new opportunities for controlling its degradation kinetics, ${ }^{14}$ release of growth factors, and new surface chemistry. ${ }^{15,16}$ Starch-compounded PCL (SPCL) combines the affinity of starch and the processability and mechanical properties of the synthetic polymer (PCL). ${ }^{17} \mathrm{Sev}$ eral experiments with SPCL have been conducted with cells, for instance, in osteoblast-like cells, ${ }^{18}$ mesenchymal stem cells, ${ }^{19,20}$ and leukocytes. ${ }^{21}$ Dynamic culture conditions ${ }^{22}$ and in vivo implantation in murine animal models ${ }^{23}$ were also explored.

Electrospinning allows for the production of polymeric ultrafine fibers with diameters ranging from a few microns to tens of nanometers. ${ }^{24}$ Because of their small diameter, polymeric nanofibers exhibit unusual properties, such as high specific surface area, flexibility in surface functionality, and superior mechanical properties. ${ }^{25}$ Additionally, nonwoven mesh structures produced by electrospinning physically mimic the structure and morphology of ECM components of a large variety of native tissues, including bone and cartilage. ${ }^{26-28}$ The aim of the present study was to assess ECM formation in PCL and SPCL electrospun nanofiber meshes, evaluating the effect of starch on the material formulation and the effect of static or dynamic culture conditions.

\section{Materials and Methods}

\section{Nanofiber meshes processing}

Polymeric solutions of PCL (PCL 787, TONE polymer, Union Carbide Chemicals and Plastics Division, Bound Brook, NJ, with $80 \mathrm{kDa}$ ) and SPCL (30\% starch, 70\% PCL; Novamont: SPCL, Mater-BI ZI01U, Novamont, Novara, Italy) were prepared by dissolving the polymer or the blend into an organic solvent mixture of chloroform:dimethylformamide 70:30 (Sigma-Aldrich, Dorset, U.K.), at concentrations of 17\% and $24 \%(\mathrm{w} / \mathrm{v})$, respectively. Polymeric solutions were placed into syringes with blunted metallic needles with an internal diameter of $0.8 \mathrm{~mm}$ attached. The syringe was coupled to a syringe pump (model KDS100, KD Scientific, Holliston, MA) to control the solution flow rate. A high-voltage power supply $(0-25 \mathrm{kV})$ was applied to the needle to generate the electric field. Aluminum foil connected to a ground was used as the fiber mesh collector. The capillary tip-to-collector distance and the flow rate were fixed at $20 \mathrm{~cm}$ and $1.0 \mathrm{~mL} / \mathrm{h}$, respectively. Nanofiber mesh production lasted $1 \mathrm{~h}$ and required $1 \mathrm{~mL}$ of polymeric solution. The applied voltage was maintained at $9 \mathrm{kV}$. Nanofiber meshes were collected and cut into $1-\mathrm{cm}^{2}$ squares. Experiments were all performed at room temperature, and the conditions were optimized for the two materials.

\section{Isolation of bovine articular chondrocytes and culture on nanofiber meshes}

Isolation and expansion of bovine articular chondrocytes (BACs) was performed according to a method previously reported..$^{29}$ Cells were cultured with expansion medium: high-glucose Dulbecco's modified Eagle medium (Sigma D-5671), containing $10 \mathrm{mM}$ of 4-(2-hydroxyethyl)1-piperazineethanesulfonic acid buffer (Sigma H-0887), L-alanyl-L-glutamine (Sigma G-8541), nonessential amino acids (Sigma M-7145), 10,000 units/mL of penicillin, 10,000 $\mu \mathrm{g} / \mathrm{mL}$ of streptomycin (Sigma P-0781), 10\% fetal calf serum (Biosera S1810, East Sussex, UK), and $10 \mathrm{ng} / \mathrm{mL}$ of basic fibroblast growth factor (bFGF) (PeproTech 100-18B, Rocky Hill, NY). Dynamic cell seeding was performed using Petri dishes. Three nanofiber meshes were placed in each Petri dish and cells suspension added to have $6.5 \times 10^{6}$ cells per nanofiber mesh. We used 20 nanofiber meshes per experimental condition for each material. Petri dishes were placed in a rotator, inside the incubator, at $60 \mathrm{rpm}$ for $72 \mathrm{~h}$.

After $72 \mathrm{~h}$, cell seeding was complete, and half of the constructs were kept in the rotator (dynamic conditions), whereas the others were placed in the incubator (static conditions). Differentiation medium (expansion medium without bFGF and with $1 \mathrm{mg} / \mathrm{mL}$ of insulin (Sigma Co.) and $1 \mathrm{mg} / \mathrm{mL}$ of ascorbic acid, Sigma A-4544) was changed every 3 days. Samples were taken at 1,2,3, and 4 weeks of culture.

\section{Scanning electron microscopy}

For scanning electron microscopy (SEM) analysis, nanofiber meshes were collected at 1, 2, 3, and 4 weeks; washed in sterile phosphate buffered saline (PBS); and immersed in 3\% glutaraldehyde (Sigma G-5882) with $0.1 \mathrm{M}$ cacodylate buffer at pH 7.4 (AGAR R1103, Essex, England) at room temperature for 1 hour. Afterwards, they were washed in PBS, osmium tetra oxide was added, and they were left for $2 \mathrm{~h}$. Finally, samples were dehydrated in crescent alcohol concentrations and left to dry. SEM micrographs were taken at different time periods and with several magnifications.

\section{Histological analysis}

Samples were collected at the end of the experiment, placed in optimal cutting temperature compound (BDH, Gurr, Lutterworth, Leicestershire, England), and stored at $-20^{\circ} \mathrm{C}$. Sections were cut $8 \mu \mathrm{m}$ thick, placed in microscopy slides, fixed for $30 \mathrm{~min}$ at $4{ }^{\circ} \mathrm{C}$ in a fresh $4 \%$ paraformaldehyde (Sigma $\mathrm{P}$-6148) solution in PBS buffer, washed twice in distilled water, and left overnight to air-dry. Slides were stored at $4{ }^{\circ} \mathrm{C}$ until they were used for staining procedures. Hematoxylin and eosin (H\&E) staining was conducted in an automatic machine (Fume Cupboard; X219/E11/LEV1, UK). Sections were washed in running water for $5 \mathrm{~min}$ and then dipped in $1 \%$ acid alcohol for 5 to $10 \mathrm{~s}$. They were washed again in water and stained in eosin for $10 \mathrm{~min}$. Another washing with water was performed, for $5 \mathrm{~min}$, after sections were dehydrated through crescent concentrations of alcohol, cleared in xylene, and mounted in dinbutyphthalate and xylene (DPX) (BDH 36029 2F). Toluidine blue staining was also performed. Staining solution was prepared by adding $1 \%$ of toluidine blue (Sigma $\mathrm{T}-0394$ ) dissolved in distilled water containing $0.5 \mathrm{~g}$ of sodium borate, followed by filtering. One drop of this solution was added to each section for 2 to $3 \mathrm{~s}$. Then sections were rinsed with distilled water and left to air dry. They were cleared in xylene and mounted in DPX. Alcian blue staining was performed by rinsing the sections in 3\% acetic acid and keeping them in 1\% Alcian blue solution (Sigma A-3157) for 18h. After that, the stain was poured off, and sections were counter- 
stained with aqueous neutral red (Sigma N-6634) for $1 \mathrm{~min}$. Sections were washed with water, left to dry, rinsed in absolute alcohol, cleared in xylene, and mounted in DPX.

\section{Immunolocalization of type I and II collagens}

Immunolocalization of type I and type II collagens was performed in fixed sections. Sections were pretreated in $10 \mathrm{mg} / \mathrm{mL}$ of hyaluronidase (Sigma Co.) for $30 \mathrm{~min}$ at $37^{\circ} \mathrm{C}$ and in $2 \mathrm{mg} / \mathrm{mL}$ of pronase (Fluka/Sigma Co.) for $30 \mathrm{~min}$ at $37^{\circ} \mathrm{C}$. Sections were washed in PBS, and endogenous peroxidase activity was quenched with $3 \%$ hydrogen peroxide in 50\% methanol (BDH 101586 6B) for $5 \mathrm{~min}$. Sections were washed in Tris-buffered saline (TBS) and blocked with $3 \%$ bovine serum albumin (Sigma A-2153) in TBS/Tween 20 (Sigma Ultra P-7949) for $1 \mathrm{~h}$ to avoid nonspecific staining. Sections were further incubated with primary antibodies (collagen type I and collagen type II;goat antitype I collagen UNLB 1310-01 and Goat antitype II collagen UNLB 1320-01) overnight at $4{ }^{\circ} \mathrm{C}$, in a humidified atmosphere. Then sections were washed once with high-salt wash solution and twice in TBS/Tween 20 for $10 \mathrm{~min}$ each and then incubated with secondary antibody from the kit for $1 \mathrm{~h}$ at room temperature, again in a humidified atmosphere. The remaining protocol is as described in the Vectastain Elite ABC Kit PK-6105 (Vector Laboratories Ltd, Peterborough, UK) and in the Vector DAB Kit (Vector Laboratories Ltd). Slides were washed in water for $5 \mathrm{~min}$ and then counterstained with hematoxylin for nuclei visualization. Finally, slides were mounted in DPX. Controls were performed using normal goat serum instead of primary antibodies, which was also included in the kit.

\section{Dimethylmethylene blue assay for glycosaminoglycan quantification}

Samples were collected at 4 weeks of culture, freeze-dried overnight, and then digested. Digestion solution was prepared by adding papain (Sigma, P-4762) and N-acetyl cysteine (Sigma A-8199) at concentrations of $0.05 \%$ and $0.096 \%$, respectively, to $50 \mathrm{~mL}$ of digestion buffer $(200 \mathrm{mM}$ of phosphate buffer containing $1 \mathrm{mM}$ ethylenediaminetetraacetic acid (Sigma E-5134), pH 6.8). Samples were incubated with $600 \mu \mathrm{L}$ of the solution overnight at $60^{\circ} \mathrm{C}$. Afterwards, they were centrifuged at $13000 \mathrm{rpm}$ for $10 \mathrm{~min}$. Supernatant was collected and stored at $-20^{\circ} \mathrm{C}$ until the assay was performed. Dimethymethylene blue (DMB) stock solution was prepared by dissolving $16 \mathrm{mg}$ of DMB powder in $900 \mathrm{~mL}$ of distilled water containing $3.04 \mathrm{~g}$ of glycine and $2.73 \mathrm{~g}$ of sodium chlo- ride. $\mathrm{pH}$ was adjusted to 3.0 with hydrochloric acid and volume to $1 \mathrm{~L}$. The solution was stored at room temperature covered by aluminum foil. Chondroitin sulfate (Sigma C8529) solution was prepared in water in a $5-\mathrm{mg} / \mathrm{mL}$ stock solution and kept refrigerated. Dilutions of this solution were made to make a standard curve. Samples were also diluted as appropriate using distilled water. Optical density was measured in a microplate reader at $530 \mathrm{~nm}$. Unseeded nanofiber meshes were used as controls and were treated in the same way as the seeded ones for the GAG assay procedure. We used three per GAG assay.

\section{Statistical analysis}

Data from triplicates of GAG quantification are presented as averages \pm standard errors. One-way analysis of variance in conjunction with Turkey's test was also performed.

\section{Results}

SEM observations of nanofiber meshes produced show a random distribution of nanofibers, as expected. This is the typical result of the electrospining process, caused by the electric field generated in the equipment (Fig. 1). PCL nanofiber meshes were composed of nanofibers with diameters in the submicron range, from 0.4 to $1.4 \mu \mathrm{m}$ (Fig. 1A). The average pore size of PCL nanofiber meshes was $72.67 \pm 31.48 \mu \mathrm{m}$. Conversely, the SPCL nanofiber meshes presented a larger dispersion of nanofiber diameters (the thinner had diameters $\sim 0.3-20 \mu \mathrm{m}$ ), probably because of the insolubility of the starch phase in the organic solvent mixture used. A PCL solution containing a suspension of colloidal starch particles was obtained, and this has important implications for fiber diameter and mesh morphology. The average pore size of SPCL, evaluated from SEM micrographs of the nanofiber meshes, was $65.21 \pm 22.07 \mu \mathrm{m}$.

SEM micrographs (Fig. 2) show that cells colonized both types of nanofiber meshes, in both culture conditions, covering their surface extensively and homogeneously. PCL nanofiber meshes keep their membrane-like shape (Fig. 2A-C, G-I), whereas SPCL nanofiber meshes tend to curl in a tubular structure (Fig. 2D-F, J-L). This structure trapped a considerable number of cells inside. Chondrocytes kept their round morphology throughout the experiment in PCL (Fig. 2A, B, G-I) and SPCL (Fig. 2E, F, K) nanofiber meshes under both culture conditions. BACs attached and spread not only to the nanofiber meshes surface, but also in the inner regions of the mesh structure, as can be observed with H\&E staining

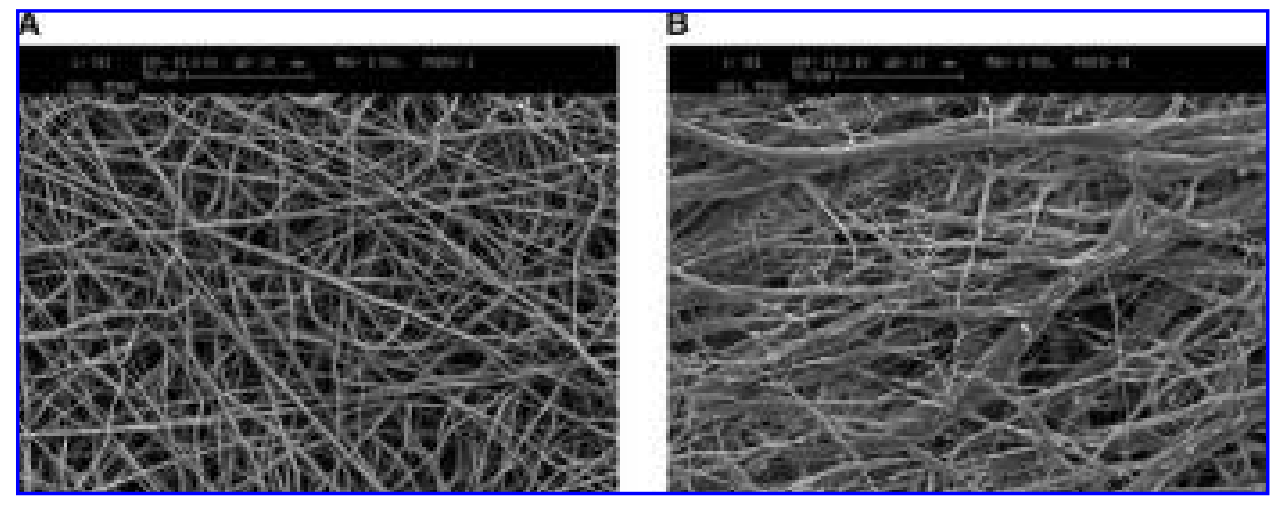

FIG. 1. Electron micrographs of (A) polycaprolactone (PCL) and (B) starch-compounded PCL nanofiber meshes processed using electrospinning. 

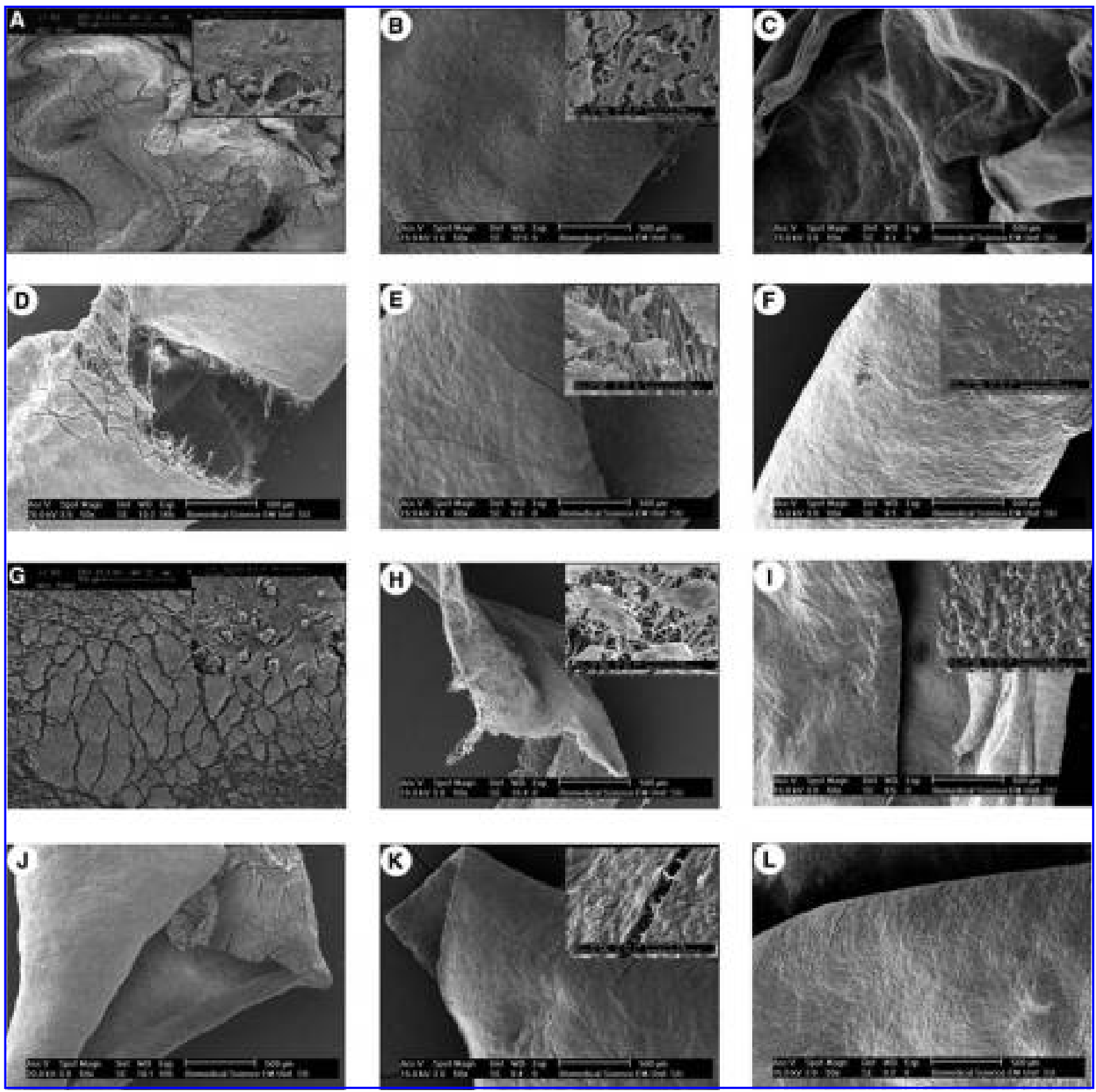

FIG. 2. Bovine articular chondrocyte (BAC) growth morphology in polycaprolactone (PCL) and starch-compounded PCL (SPCL) nanofiber meshes at different time points of the experiment (14 days (A, D, G, J), 21 days (B, E, H, K), and 28 days $(\mathbf{C}, \mathbf{F}, \mathbf{I}, \mathbf{L})$ ) under static (A-F) or dynamic (G-L) culture conditions. Different magnifications were used to highlight cell morphology. Cell growth was identical in static culture conditions for PCL (A-C) or SPCL (D-F) nanofiber meshes. The same was observed in dynamic culture conditions for PCL (G-I) and SPCL (J-L).

(Fig. 3A, D, G, J). PCL and SPCL nanofiber meshes showed similar cell distribution, as well as the presence of proteoglycans, as shown by toluidine blue (Fig. 3B, E, H, K) and Alcian blue staining (Fig. 3C, F, I, L). Darker Alcian blue staining can be seen better in histological sections from static culture conditions (Fig. 3C, F) than in dynamic conditions (Fig. 3I, L). This result indicates the presence of more sulfated proteoglycans in static conditions. SPCL nanofiber meshes curled and formed tubes, entrapping a considerable number of cells inside. There were a large amount of proteoglycans in these protected inner regions, as well as collagen type I and type II (data not shown). Both collagens types were also detected in static (Fig. 4A-F) and dynamic culture conditions (Fig. 4G-L). Collagen type I was more evident in sections prepared from SPCL nanofiber meshes (both culture conditions) (Fig. 4E, K) than in sections from PCL nanofiber meshes (Fig. 4B, H), indicating that this combination of structure and material generated a predominance of fibrocartilage. 


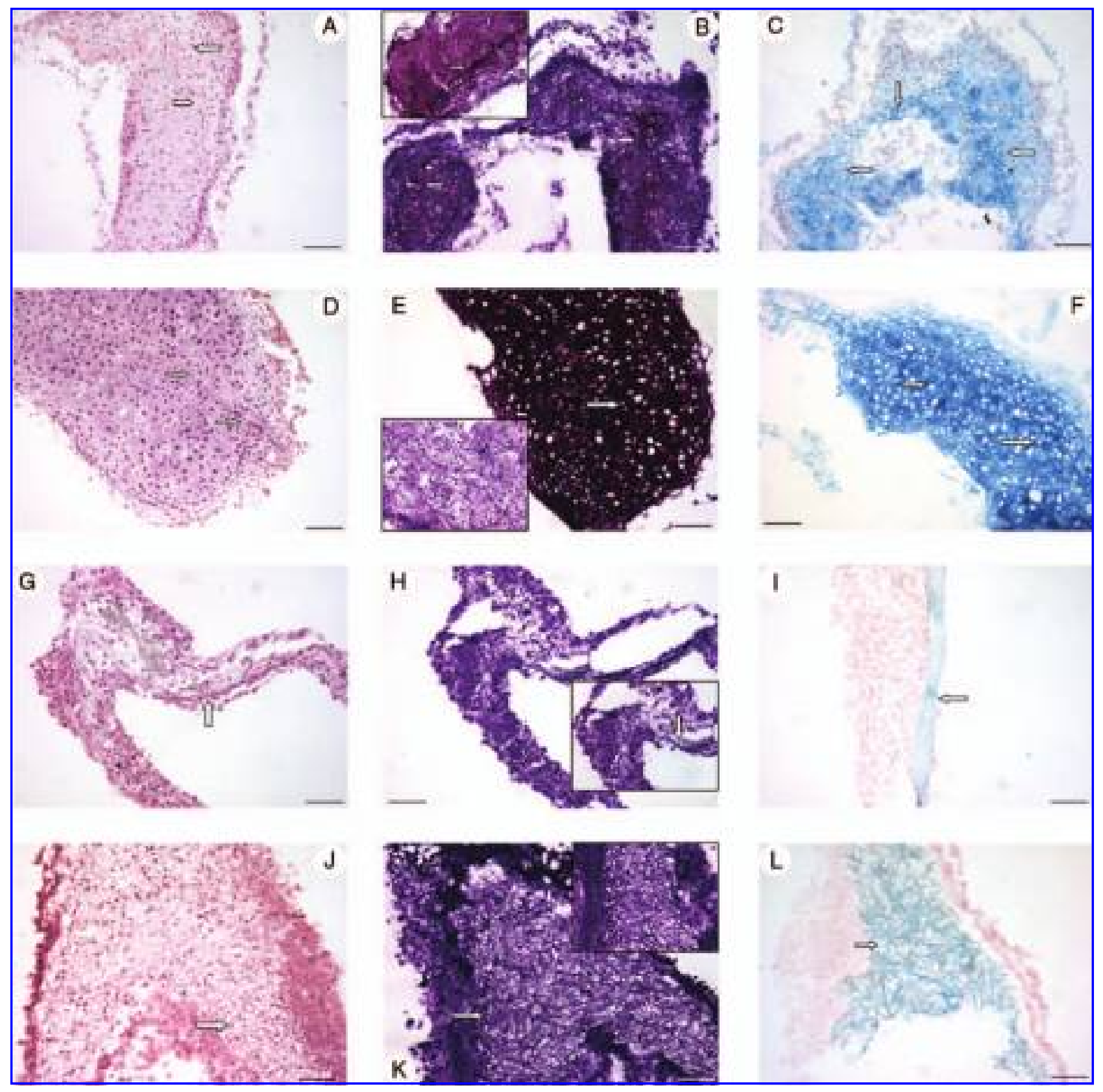

FIG. 3. Production of extracellular matrix in polycaprolactone (PCL) and starch-compounded PCL (SPCL) nanofiber meshes at 4 weeks of culture under static $(\mathbf{A}-\mathbf{F})$ and dynamic $(\mathbf{G}-\mathbf{L})$ culture conditions. Cells were able to attach to the nanofibers structure, as shown with hematoxylin and eosin staining, for PCL $(\mathbf{A}, \mathbf{G})$ and SPCL $(\mathbf{D}, \mathbf{J})$. Cells penetrated this structure and produced proteoglycans, detected using toluidine blue staining, in PCL (B, H) and SPCL (E, K) nanofiber meshes. Sulphated proteoglycans were detected using Alcian blue staining, for PCL (C, I) and SPCL (F, L) nanofiber meshes. Two magnifications (10x and 20x) were used at each staining for microscopic observation. Scale bar $=100 \mu \mathrm{m}$. Arrows highlight nanofiber mesh structure. Color images available online at www.liebertonline.com/ten.

Quantification of GAGs in PCL nanofiber meshes revealed the presence of approximately $2.0 \%$ of total GAGs in the ECM in static culture conditions, versus $0.8 \%$ of total GAGs found in dynamic conditions (Fig. 5). No statistically significant difference was found between these results $(p>0.05)$. As for the SPCL nanofiber meshes, results revealed $2.4 \%$ of total GAGs for the static cultures, versus $1.1 \%$ for the dynamic ones. Again, there was not a statistically significant difference between static and dynamic cultures $(p>0.05)$. The same lack of statistical significance was found in differences between results obtained with the two types of materials $(p>0.05)$.

\section{Discussion}

The main goal of designing and producing a new scaffold is to provide a better microenvironment for cells in an attempt to substitute the natural ECM milieu while they expand and repair the tissue. Many materials have been proposed for 


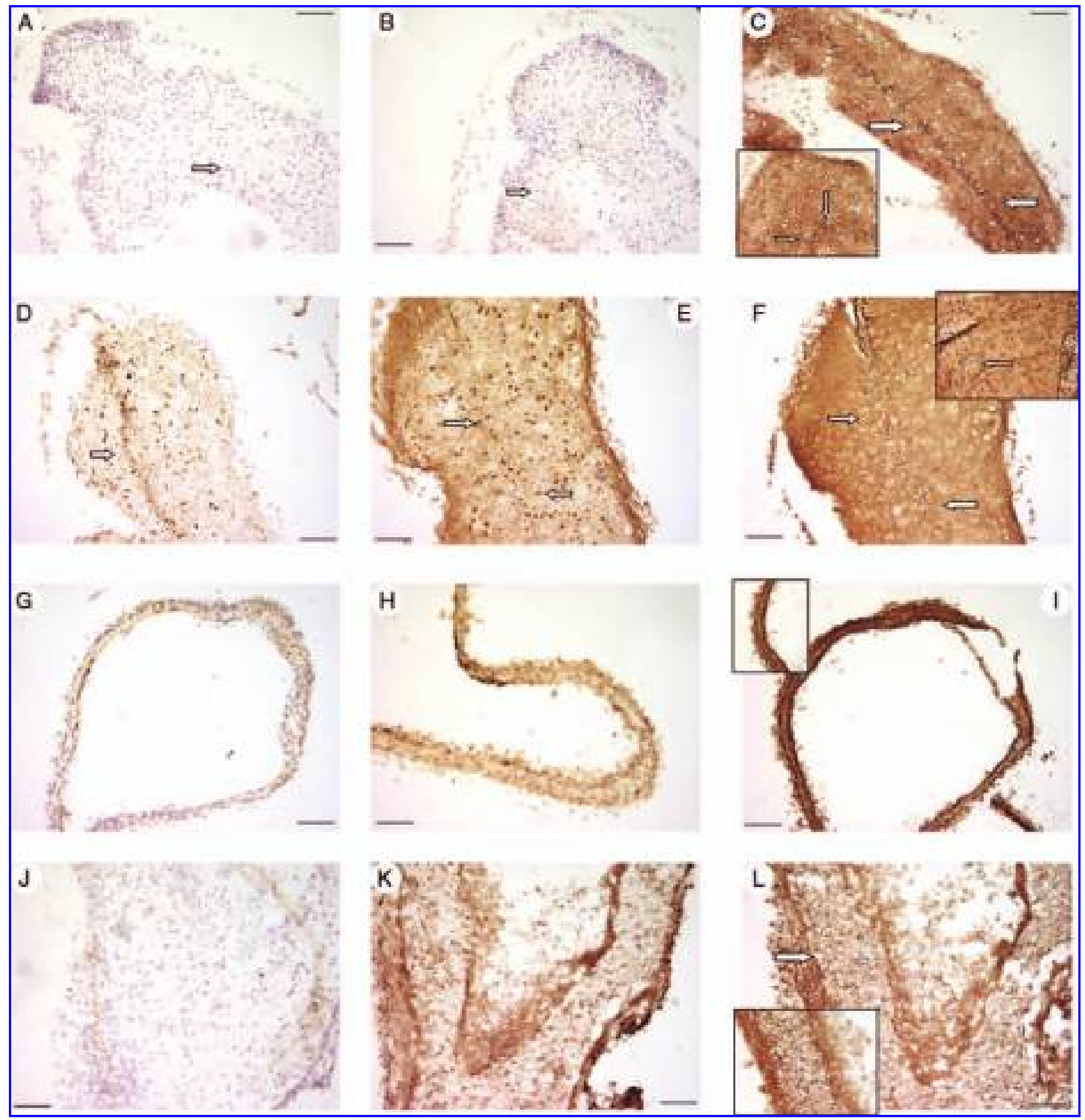

FIG. 4. Immunolocalization of collagens in polycaprolactone (PCL) and starch-compounded PCL (SPCL) nanofiber meshes at 4 weeks of culture under static $(\mathbf{A}-\mathbf{F})$ and dynamic $(\mathbf{G}-\mathbf{L})$ culture conditions. Controls $(\mathbf{A}, \mathbf{D}, \mathbf{G}, \mathbf{J})$ were performed with normal goat serum. Under static conditions, collagen type I was detected only for SPCL (E) nanofiber meshes. Collagen type II, alternatively, was detected for PCL (C) and SPCL (F) nanofiber meshes. Under dynamic conditions, collagen type I and type II were detected in PCL $(\mathbf{H}, \mathbf{I})$ and SPCL $(\mathbf{K}, \mathbf{L})$ nanofiber meshes. Two magnifications (10x and 20x) were used at each staining for microscopic observation. Scale bar $=100 \mu \mathrm{m}$. Arrows highlight nanofiber mesh structure. Color images available online at www.liebertonline.com/ten.

scaffold production. Of those, natural polymers such as starch $^{14,16-23,27,30-38}$ and biodegradable polyesters, for example, PCL, ${ }^{11,13,17,39-41}$ have been developed. In this case, an electrospinning technique was used to explore those materials and obtain a new SPCL fibrous structure. Electrospinning was used to produce meshes that were intended to provide good conditions for BACs to produce ECM. Polymer nanofibers have recently been used in the fields of biotechnology and biomedicine. ${ }^{42}$ Several tissue-engineering approaches, aiming at bone $\mathrm{e}^{11,32,43}$ and cartilage $\mathrm{e}^{40,41}$ regeneration, have also been explored. SEM micrographs confirmed that cells were able to colonize both nanofiber meshes, reinforcing the idea that PCL and SPCL are suitable polymers for the intended purpose. ${ }^{17}$ BACs attached and spread not only at the surface of the 


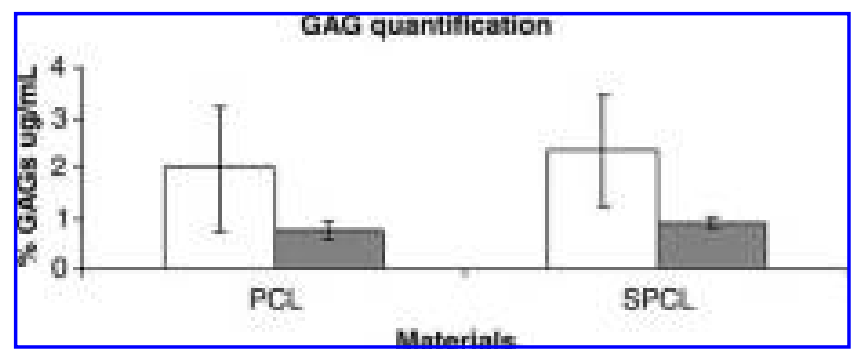

FIG. 5. Glycosaminoglycan (GAG) quantification for polycaprolactone (PCL) and starch-compounded PCL (SPCL) nanofiber meshes at 4 weeks of culture. Static culture conditions are represented in white, dynamic culture conditions in gray.

nanofiber meshes, but also in the inner regions of the mesh structure, as can be observed with H\&E staining. Our present results indicate that BACs penetrate the structure, colonizing it and using it as a support for cell activity. This is, to the best of our knowledge, an encouraging result, because most of the studies with nanofiber meshes obtained using electrospinning do not show so clear a penetration of cells into the mesh structure. ${ }^{25}$ Cells were able to produce ECM, as shown by toluidine and Alcian blue staining, which detected GAG accumulation. Collagen type I and II were both detected, expression of collagen type I being greater in SPCL meshes. Collagen type $\mathrm{I}$ is associated with fibrocartilage tissue, ${ }^{44}$ which is undesirable when articular cartilage is the goal of a regeneration strategy. PCL electrospun nanofiber meshes have been proposed as novel scaffolds for tissue engineering, ${ }^{45}$ especially for cartilage repair. Li et al. showed the production of cartilaginous matrix with fetal bovine chondrocytes seeded onto PCL nanofibrous scaffolds, highlighting the importance of these materials for proliferation and maintenance of the chondrocyte phenotype. ${ }^{41}$ Recent work from the same authors also show the versatility of these nanofibrous scaffolds in sustaining chondrogenic differentiation of human mesenchymal stem cells. ${ }^{40,46}$

GAG production was not statistically different in the two types of materials. There was a slightly difference between them, being higher in SPCL than in PCL (2.4\% vs $2.0 \%)$. SPCL is a blend composed of corn starch and polycaprolactone that has been extensively studied as a biomaterial for different biomedical applications. ${ }^{17,27,31,47}$ It was recently reported that it supports BAC adhesion, proliferation, and differentiation in SPCL fiber-based scaffolds. ${ }^{47}$ It was unexpected to find more GAGs in static cultures than in dynamic ones, because chondrocytes are naturally subjected to a fluid flow microenvironment and therefore could be stimulated by these culture conditions. ${ }^{48}$ The use of mechanical force can be employed in their culturing process to produce a phenotypically correct tissue, ${ }^{49}$ although it needs to replicate relevant physiologic conditions. Agitation was used during culture to produce a phenotipically adequate tissue, but it may be concluded that these conditions were not sufficient. We can hypothesize that the higher GAG content in static cultures observed in this study was due to some GAGs being released into the culture medium during the experiment when the culture medium was mixed. Furthermore, seeding in spinner flasks may have somehow caused some cell damage, due to the turbulent flow generated by the magnetic stirring. ${ }^{50}$ Similar results were obtained with bovine calf chondrocytes seeded onto fibrous polyglycolic acid meshes, where constructs exposed to similar mixing synthesized and released more GAGs into the culture medium and resulted in lower fractions of GAGs. ${ }^{51}$ Another study showed that mixing may increase the loss of GAGs from the construct, decreasing pericellular GAG concentration. ${ }^{52}$

Data did not allow us to substantiate clearly which of the materials is best for the approach used in this work. It is known that a suitable scaffold for tissue engineering must have good surface properties, which will allow cell attachment and efficient transport of nutrients to growing cells. In that sense, a highly hydrophilic material would allow greater water uptake and, consequently, a greater nutrient supply to cells. ${ }^{53,54}$ PCL is more hydrophobic, whereas SPCL is more hydrophilic, which is more advantageous for cell growth than PCL. PCL is a homopolymer with slow degradation kinetics. It is in clinical use for many applications and could be the biodegradable material of choice, although a wide range of options for the development of PCL has already been explored. One additional alternative to develop its performance being proposed herein is to compound it with other biodegradable materials, those compounds offering a new range of tunable properties. If we accept that there are advantages to working with a natural-based polymer, the combination of starch with PCL may lead to an attractive combination of properties that may be tailored to specific applications, including cartilage tissue engineering. This material has been extensively studied for biomedical applications because of its biocompatibility and biodegradability ${ }^{14,16,18,27,33,55}$ and potential of inducing low levels of inflammatory response. ${ }^{21}$ Its degradation kinetics can be controlled using material composition, having a more hydrophilic character and enabling the design of controlled-release systems for growth factors. ${ }^{56}$ Results indicate that SPCL may be slightly more effective in sustaining ECM production, but it was also shown that greater expression of collagen type I may be detrimental in obtaining hyaline cartilage from BACs cultured in these meshes. Further optimization of this material will be addressed to overcome this limitation.

\section{Conclusion}

The aim of this work was to determine whether a blend of starch with PCL (SPCL) would have similar performance in sustaining BAC growth and ECM production as PCL. PCL and SPCL electrospun nanofiber meshes were both interesting structures for cartilage tissue-engineering strategies. PCL and SPCL nanofiber meshes seeded dynamically evidenced a good response for this purpose. An interesting result was the evidence that chondrocytes proliferated in external regions and showed some capacity to migrate into inner regions of both types of fiber meshes. BACs were able to produce ECM under static or dynamic culture conditions. Static culture conditions lead to enhanced ECM production. These observations were confirmed by staining of proteoglycans, immunolocalization of collagens type I and type II, and GAG detection.

In this work, we confirmed evidence from the literature that sustain the claim that PCL nanofiber meshes are suitable for tissue-engineering approaches and also propose a new scaffold for cartilage tissue engineering (SPCL 
nanofiber meshes). The data did not show obvious qualitative or quantitative differences between the two materials; nevertheless, we consider it advantageous to use SPCL, not only because of its natural-based composition, but also because it allows for greater control of the degradation kinetics and further functionalization of its structure. It is our understanding that both materials have a role to play in cartilage regeneration strategies and that, in particular, SPCL nanofiber meshes can be further enhanced, not only in terms of degradability, but also by providing opportunities for local release of growth factors for articular cartilage tissueengineering strategies.

\section{Acknowledgments}

M. Alves da Silva would like to acknowledge the Portuguese Foundation for Science and Technology (FCT) for her grant (SFRH/BD/28708/2006), Marie Curie ActionsALEA JACTA EST (MEST-CT-2004-008104), European NoE EXPERTISSUES (NMP3-CT-2004-500283), IP GENOSTEM (LSHB-CT-2003-503161) and NATURALLY NANO (POCTI/ EME/58982/2004).

\section{References}

1. Brandt, K.D. The role of analgesics in the management of osteoarthritis pain. Am J Ther 7, 75, 2000.

2. Ringe, J., Haupl, T., and Sittinger, M. Future of tissue engineering in rheumatic diseases. Expert Opin Biol Ther 7, 283, 2007.

3. Hoemann, C.D., Sun, J., Legare, A., McKee, M.D., and Buschmann, M.D. Tissue engineering of cartilage using an injectable and adhesive chitosan-based cell-delivery vehicle. Osteoarthritis Cartilage 13, 318, 2005.

4. Martin, I., Miot, S., Barbero, A., Jakob, M., and Wendt, D. Osteochondral tissue engineering. J Biomech 40, 750, 2007.

5. Freed, L.E., Martin, I., and Vunjak-Novakovic, G. Frontiers in tissue engineering - in vitro modulation of chondrogenesis. Clin Orthop Relat Res 367S, S46 1999.

6. Martin, I., Wendt, D., and Hereber, M. The role of bioreactors in tissue engineering. Trends Biotechnol 22, 802004.

7. Crawford, A., Frazer, A., Dickinson, S., Sims, T., Hollander, A.P., and Hatton, P.V. Effect of biomaterial morphology on the deposition of collagen in tissue engineered cartilage constructs. Sydney, Australia: Transactions-7th World Biomaterials Congress, 2004, p. 594.

8. Hatton, P.V., Crawford, A., Frazer, A., Fraser, S., Dickinson, S., and Hollander, A.P. Effect of culture conditions and cell source on the quality of tissue engineered cartilage constructs. Sydney, Australia: Transactions-7th World Biomaterials Congress, 2004, p. 1621.

9. Agrawal, C., and Ray, R. Biodegradable polymeric scaffolds for musculoskeletal tissue engineering. J Biomed Mater Res 55, 141, 2001.

10. Azevedo, H., Gomes, M.E., Malafaya, P.B., Marques, A.P., Salgado, A.J., and Reis, R.L. Natural Origin Degradable Polymers in Biomedical Applications. CA: American Scientific Publishers, Valencia, CA, 2006.

11. Yoshimoto, H., Shin, Y.M., Terai, H., and Vacanti, J.P. A biodegradable nanofiber scaffold by electrospinning and its potential for bone tissue engineering. Biomaterials 24, 2077, 2003.

12. Venugopal, J., Ma, L.L., Yong, T., and Ramakrishna, S. In vitro study of smooth muscle cells on polycaprolactone and collagen nanofibrous matrices. Cell Biol Int 29, 861, 2005.
13. Zhang, Y.Z., Venugopal, J., Huang, Z.M., Lim, C.T., and Ramakrishna, S. Characterization of the surface biocompatibility of the electrospun PCL-collagen nanofibers using fibroblasts. Biomacromolecules 6, 2583, 2005.

14. Azevedo, H.S., Gama, F.M., and Reis, R.L. In vitro assessment of the enzymatic degradation of several starch based biomaterials. Biomacromolecules 4, 1703, 2003.

15. Alves, C.M., Yang, Y., Carnes, D.L., Ong, J.L., Sylvia, V.L., Dean, D.D., Agrawal, C.M., and Reis, R.L. Modulating bone cells response onto starch-based biomaterials by surface plasma treatment and protein adsorption. Biomaterials 28, 307, 2007.

16. Costa, S.A., and Reis, R.L. Immobilisation of catalase on the surface of biodegradable starch-based polymers as a way to change its surface characteristics. J Mater Sci Mater Med 15, 335, 2004.

17. Wang, Y., Rodriguez-Perez, M.A., Reis, R.L., and Mano, J.F. Thermal and thermomechanical behaviour of polycaprolactone and starch/polycaprolactone blends for biomedical applications. Macromol Mater Eng 290, 792, 2005.

18. Marques, A.P., Cruz, H.R., Coutinho, O.P., and Reis, R.L. Effect of starch-based biomaterials on the in vitro proliferation and viability of osteoblast-like cells. J Mater Sci Mater Med 16, 833, 2005.

19. Gomes, M.E., Holtorf, H.L., Reis, R.L., and Mikos, A.G. Influence of the porosity of starch-based fiber mesh scaffolds on the proliferation and osteogenic differentiation of bone marrow stromal cells cultured in a flow perfusion bioreactor. Tissue Eng 12, 801, 2006.

20. Gomes, M.E., Sikavitsas, V.I., Behravesh, E., Reis, R.L., and Mikos, A.G. Effect of flow perfusion on the osteogenic differentiation of bone marrow stromal cells cultured on starchbased three-dimensional scaffolds. J Biomed Mater Res A 67, 87, 2003.

21. Marques, A.P., Reis, R.L., and Hunt, J.A. The effect of starchbased biomaterials on leukocyte adhesion and activation in vitro. J Mater Sci Mater Med 16, 1029, 2005.

22. Gomes, M.E., Bossano, C.M., Johnston, C.M., Reis, R.L., and Mikos, A.G. In vitro localization of bone growth factors in constructs of biodegradable scaffolds seeded with marrow stromal cells and cultured in a flow perfusion bioreactor. Tissue Eng 12, 177, 2006.

23. Marques, A.P., Reis, R.L., and Hunt, J.A. An in vivo study of the host response to starch-based polymers and composites subcutaneously implanted in rats. Macromol Biosci 5, 775, 2005.

24. Reneker, D., and Chun, I. Nanometre diameter fibers of polymer, produced by electrospinning. Nanotechnology 7, 216, 1996.

25. Huang, Z.-M., Zhang, Y.-Z., Kotaki, M., and Ramakrishna, S. A review on polymer nanofibers by electrospinning and their applications in nanocomposites. Composites Sci Technol 63, 2223, 2003.

26. Ma, Z., Kotaki, M., Inai, R., and Ramakrishna, S. Potential of nanofiber matrix as tissue-engineering scaffolds. Tissue Eng 11, 101, 2005.

27. Oliveira, A.L., and Reis, R.L. Pre-mineralisation of starch/ polycrapolactone bone tissue engineering scaffolds by a calcium-silicate-based process. J Mater Sci Mater Med 15, 533, 2004.

28. Smith, L., and Ma, P. Nano-fibrous scaffolds for tissue engineering. Colloids Surf B Biointerfaces 39, 125, 2004.

29. Crawford, A., and Dickinson, S.C. Chondrocyte Isolation, Expansion and Culture on Polymer Scaffolds. Totowa, NJ: Humana Press Inc., 2004. 
30. Salgado, A.J., Coutinho, O.P., and Reis, R.L. Novel starchbased scaffolds for bone tissue engineering: cytotoxicity, cell culture, and protein expression. Tissue Eng 10, 465, 2004.

31. Santos, M.I., Fuchs, S., Gomes, M.E., Unger, R.E., Reis, R.L., and Kirkpatrick, C.J. Response of micro- and macrovascular endothelial cells to starch-based fiber meshes for bone tissue engineering. Biomaterials 28, 240, 2006.

32. Tuzlakoglu, K., Bolgen, N., Salgado, A.J., Gomes, M.E., Piskin, E., and Reis, R.L. Nano- and micro-fiber combined scaffolds: a new architecture for bone tissue engineering. J Mater Sci Mater Med 16, 1099, 2005.

33. Gomes, M.E., Reis, R.L., Cunha, A.M., Blitterswijk, C.A., and de Bruijn, J.D. Cytocompatibility and response of osteoblasticlike cells to starch-based polymers: effect of several additives and processing conditions. Biomaterials 22, 1911, 2001.

34. Marques, A.P., Reis, R.L., and Hunt, J.A. Cytokine secretion from mononuclear cells cultured in vitro with starch-based polymers and poly-L-lactide. J Biomed Mater Res A 71, 419, 2004.

35. Marques, A.P., Reis, R.L., and Hunt, J.A. Evaluation of the potential of starch-based biodegradable polymers in the activation of human inflammatory cells. J Mater Sci Mater Med 14, 167, 2003.

36. Marques, A.P., Reis, R.L., and Hunt, J.A. The biocompatibility of novel starch-based polymers and composites: in vitro studies. Biomaterials 23, 1471, 2002.

37. Alves, C.M., Reis, R.L., and Hunt, J.A. Preliminary study on human protein adsorption and leukocyte adhesion to starchbased biomaterials. J Mater Sci Mater Med 14, 157, 2003.

38. Pashkuleva, I., Marques, A.P., Vaz, F., and Reis, R.L. Surface modification of starch based blends using potassium permanganate-nitric acid system and its effect on the adhesion and proliferation of osteoblast-like cells. J Mater Sci Mater Med 16, 81, 2005.

39. Shao, X.X., Hutmacher, D.W., Ho, S.T., Goh, J.C.H., and Lee, E.H. Evaluation of a hybrid scaffold/cell construct in repair of high-load-bearing osteochondral defects in rabbits. Biomaterials 27, 10712006.

40. Li, W.-J., Tuli, R., Okafor, C., Derfoul, A., Danielson, K.G., Hall, D.J., and Tuan, R.S. A three-dimensional nanofibrous scaffold for cartilage tissue engineering using human mesenchymal stem cells. Biomaterials 26, 599 - 609, 2005.

41. Li, W.-J., Danielson, K.G., Alexander, P.G., and Tuan, R.S. Biological response of chondrocytes cultured in threedimensional nanofibrous poly(-caprolactone) scaffolds. I Biomed Mater Res 67, 1105, 2003.

42. Venugopal, J., and Ramakrishna, S. Applications of polymer nanofibers in biomedicine and biotechnology. Appl Biochem Biotechnol 125, 147, 2005.

43. Santos, M.I., Fuchs, S., Gomes, M.E., Unger, R.E., Reis, R.L., and Kirkpatrick, C.J. Response of micro- and macrovascular endothelial cells to starch-based fiber meshes for bone tissue engineering. Biomaterials 28, 240, 2007.

44. Freemont, A.J., and Hoyland, J. Lineage plasticity and cell biology of fibrocartilage and hyaline cartilage: Its significance in cartilage repair and replacement. Eur J Radiol 57, $32,2006$.

45. Li, W.-J., Laurencin, C., Caterson, E., Tuan, R.S., and Ko, F.K. Electrospun nanofibrous structure: A novel scaffold for tissue engineering. J Biomed Mater Res 60, 613, 2002.

46. Li, W.-J., Tuli, R., Huang, X., Laquerriere, P., and Tuan, R.S. Multilineage differentiation of human mesenchymal stem cells in three-dimensional nanofibrous scaffold. Biomaterials 26, 5158, 2005.

47. Oliveira, J.T., Crawford, A., Mundy, J.M., Moreira, A.R., Gomes, M.E., Hatton, P.V., and Reis, R.L. A cartilage tissue engineering approach combining starch-polycaprolactone fibre mesh scaffolds with bovine articular chondrocytes. J Mater Sci Mater Med 18, 295, 2007.

48. Gaissmaier, C., Fritz, J., Krackhardt, T., Flesch, I., Aicher, W.K., and Ashammakhi, N. Effect of human platelet supernatant on proliferation and matrix synthesis of human articular chondrocytes in monolayer and three-dimensional alginate cultures. Biomaterials 26, 2005.

49. Darling, E.M., and Athanasiou, K. Articular cartilage bioreactors and bioprocesses. Tissue Eng 9, 9, 2003.

50. Vunjak-Novakovic, G., Martin, I., Obradovic, B., Treppo, S., Grodzinsky, A.J., Langer, R., and Freed, L.E. Bioreactor cultivation conditions modulate the composition and mechanical properties of tissue-engineered cartilage. I Orthop Res 17, 130, 1999.

51. Gooch, K.J., Kwon, J.H., Blunk, T., Langer, R., Freed, L.E., and Vunjak-Novakovic, G. Effects of mixing intensity on tissue-engineered cartilage. Biotechnol Bioeng 72, 402, 2001.

52. Sah, R.L., Grodzinsky, A.J., Plaas, A.H., and Sandy, J.D. Effects of tissue compression on the hyaluronate-binding properties of newly synthesized proteoglycans in cartilage explants. Biochem J 267, 803, 1990.

53. Ivirico, J.L., Martinez, E.C., Sanchez, M.S., Criado, I.M., Ribelles, J.L., and Pradas, M.M. Structure and properties of methacrylate-endcapped caprolactone networks with modulated water uptake for biomedical applications. J Biomed Mater Res B Appl Biomater 83, 266, 2007.

54. Hutcheon, G.A., Messiou, C., Wyre, R.M., Davies, M.C., and Downes, S. Water absorption and surface properties of novel poly(ethylmethacrylate) polymer systems for use in bone and cartilage repair. Biomaterials 22, 667, 2001.

55. Mendes, S.C., Reis, R.L., Bovell, Y.P., Cunha, A.M., van Blitterswijk, C.A., and de Bruijn, J.D. Biocompatibility testing of novel starch-based materials with potential application in orthopaedic surgery: a preliminary study. Biomaterials 22, 2057, 2001.

56. Malafaya, P.B., Elvira, C., Gallardo, A., San Roman, J., and Reis, R.L. Porous starch-based drug delivery systems processed by a microwave route. J Biomater Sci Polym Ed 12, 1227, 2001.

Address reprint requests to: Marta Alves da Silva, M.Sc. 3B's Research Group Department of Polymer Engineering University of Minho Avepark

Zona Industrial da Gandra

S. Cláudio do Barco 4806-909 Caldas das Taipas

Guimarães Portugal

E-mail: msilva@dep.uminho.pt

Received: October 8, 2007 Accepted: May 19, 2008 